Diagnostic yields were as follows; sputum smear 3\% and culture $18 \%$; pleural fluid smear 0 and culture $36 \%$; pleural biopsy smear $11 \%$ and culture 54\%; pleural biopsy histology 93\%. Culture yield for pleural fluid and biopsy was $61 \%$, and overall culture yield for sputum, pleural fluid and biopsy was $68 \%$.

All patients' received quadruple TB therapy, with $82 \%$ of patients being given the standard six-month therapy. 92\% showed an excellent radiological response, with the x-ray being normal, or with only minor residual abnormalities. To the present date, there has been no diagnosed recurrence of TB.

Conclusions Pleural TB contributes significantly to the overall burden of pleural disease in this London hospital. TB should be considered in patients presenting with pleural disease, especially young patients from ethnic minority backgrounds. To improve the diagnosis and treatment of pleural TB, culture yields need improvement.

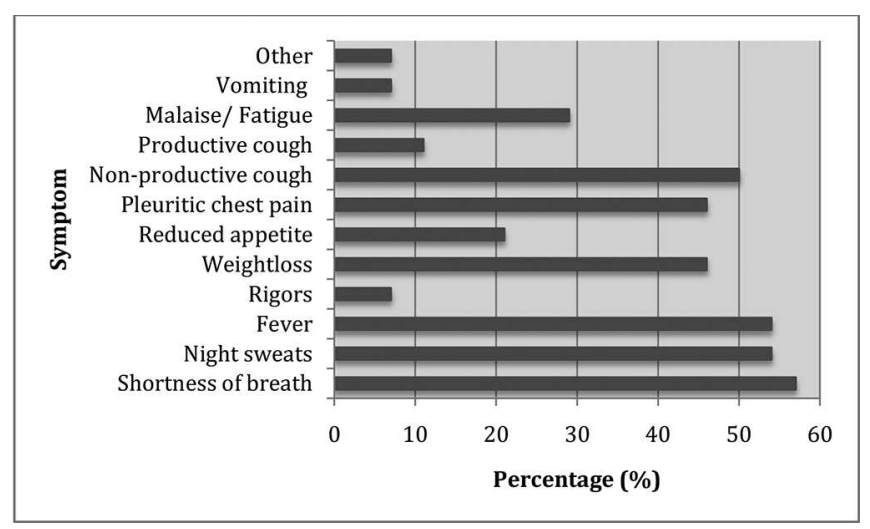

Abstract P222 Figure 1. Percentage of patients presenting with various symptoms.

\section{P223 MEDICAL THORACOSCOPY - PATIENT EXPERIENCE OF ADVANCED NURSE PRACTITIONER- PROVIDED CONSCIOUS SEDATION}

D O'Neill, K Pink, AA lonescu; Royal Gwent Hospital - Aneurin Bevan HB, Newport, UK

\subsection{6/thoraxjnl-2013-204457.375}

Medical thoracoscopy (MT) is a safe procedure provided by respiratory physicians across the UK. The conscious sedation is administered by staff with a variable level of training.

Aim To assess a simple patient comfort score at MT when conscious sedation (CS) was provided by an advanced nurse practitioner (ANP) compared to others (senior nurse, endoscopy nurse or junior doctor).

Methods A patient comfort score is recorded routinely as part of our thoracoscopy service immediately after the procedure once the patient is awake in recovery. The ANP is an ALS provider with senior experience in critical care. Intraoperative administration of midazolam for sedation and alfentanyl for pain control pre-biopsies was undertaken by a dosing schedule determined by the level of sedation assessed by the ANP who also monitored the patient during the procedure. When CS was given by others midazolam was administered in an initial bolus followed by boluses as indicated by the thoracoscopist in keeping with information by the monitoring nurse on the level of sedation or discomfort. Patient comfort score was evaluated using a 5 point scale within 30 minutes of return to the recovery area. The CS was administered either by the ANP or others in keeping with their availability on the day; no randomisation was performed. The analysis used SPSS programme.

Results 50 consecutive patients undergoing thoracoscopy were included. 27 had CS by ANP (group 1) and 23 by others (group 2). Overall the procedure was well tolerated. Patient comfort score was better in group 1 (mean, SD $0.59+/-0.8$ ) vs. group 2 $(1.63+/-1.3), \mathrm{p}<0.05$. This was achieved with a larger dose of midazolam in group $1(2.87+/-1.12 \mathrm{mg})$ vs. group $2(2.30$ $+/-0.70 \mathrm{mg}), \mathrm{p}<0.05$ and smaller dose of alfentanyl $(0.245$ $+/-0.14 \mathrm{mg})$ in group 1 vs. group $2(0.527+/-0.25 \mathrm{mg}), \mathrm{p}<$ 0.01 .

Conclusions conscious sedation for medical thoracoscopy when provided by a critical care experienced ANP resulted in an improved patient experience of the procedure and this was achieved through and adequate use of midazolam and lesser doses of alfentanyl; this was cost-saving since the ANP also monitored the patient. Retaining of trained staff is essential for this specialised service.

\section{Inhaled therapy in COPD}

\section{P224 EVALUATION OF INHALED CORTICOSTEROID RELATED PNEUMONIA MORTALITY IN PATIENTS WITH COPD WHO WOULD NOT FIT THE CRITERIA FOR INCLUSION IN RANDOMISED CONTROLLED TRIALS}

${ }^{1}$ A Singanayagam, ${ }^{2} \mathrm{~S}$ Schembri, ${ }^{3} \mathrm{AR}$ Akram, ${ }^{2} \mathrm{R}$ Archibald, ${ }^{2} \mathrm{~L}$ Peet, ${ }^{3} \mathrm{G}$ Fleming, ${ }^{3} \mathrm{~J}$ Taylor, ${ }^{4} \mathrm{P}$ Williamson, ${ }^{2} \mathrm{P}$ Short, ${ }^{3} \mathrm{~J}$ Chalmers; 'St Mary's Hospital, London, United Kingdom; ${ }^{2}$ Ninewells Hospital, Dundee, United Kingdom; ${ }^{3}$ Royal Infirmary of Edinburgh, Edinburgh, United Kingdom; ${ }^{4}$ Perth Royal Infirmary, Perth, United Kingdom

\subsection{6/thoraxjnl-2013-204457.376}

Background Large randomised controlled trials such as TORCH (towards a revolution in chronic obstructive pulmonary disease (COPD) health) report an increased risk of pneumonia associated with use of inhaled corticosteroids (ICS) in COPD but no corresponding increase in pneumonia-related mortality. However, these trials exclude patients who are elderly, comorbid, have co-existing lung conditions or use long-term oxygen therapy and may not be representative of 'real-world' practice. We hypothesised that ICS use in patients that are ineligible for TORCH would be associated with increased risk of pneumonia hospitalisations and mortality.

Methods We carried out an analysis of 2 independent cohorts. The EXODUS cohort included patients admitted with COPD exacerbation and considered outcomes over 1 year including pneumonia hospitalisations and pneumonia-related mortality. The Edinburgh pneumonia study included patients hospitalised with community-acquired pneumonia with the primary outcome of 30-day mortality. A secondary analysis of patients from this cohort with spirometry-confirmed COPD during clinical stability was conducted.

Results There were 977 patients included from the EXODUS cohort. 106 patients (10.8\%) were hospitalised for pneumonia and 18 patients $(1.8 \%)$ had pneumonia-related mortality within 12 months of initial admission. 497 patients (50.9\%) would have been ineligible for the TORCH study. In a Cox proportional hazards model, adjusting for relevant confounders, patients who were ineligible for TORCH had an increased risk of pneumonia hospitalisation (HR 1.60; 95\% CI 1.04-2.45) and an increased risk of pneumonia-related mortality (HR 6.1; 95\% CI 1.7-22.0). Figure 1 shows a cox adjusted survival curve for 
pneumonia hospitalisations in patients eligible and ineligible for TORCH.

There were 376 patients with COPD included from the Edinburgh pneumonia study. The 30 -day mortality rate was $12.0 \%$. 186 patients $(38.0 \%)$ would have been ineligible for TORCH. After adjustment for relevant confounders, ICS use in patients classified as ineligible for TORCH was associated with increased risk of 30-day mortality (HR 1.85 95\% CI 1.00-2.41).

Conclusion Patients ineligible for RCTs such as TORCH are at increased risk of ICS related pneumonia mortality and hospitalisation. Existing studies may therefore underestimate the true impact of ICS related pneumonia in the "real-world" setting.

\section{Pneumonia related hospitalisations}

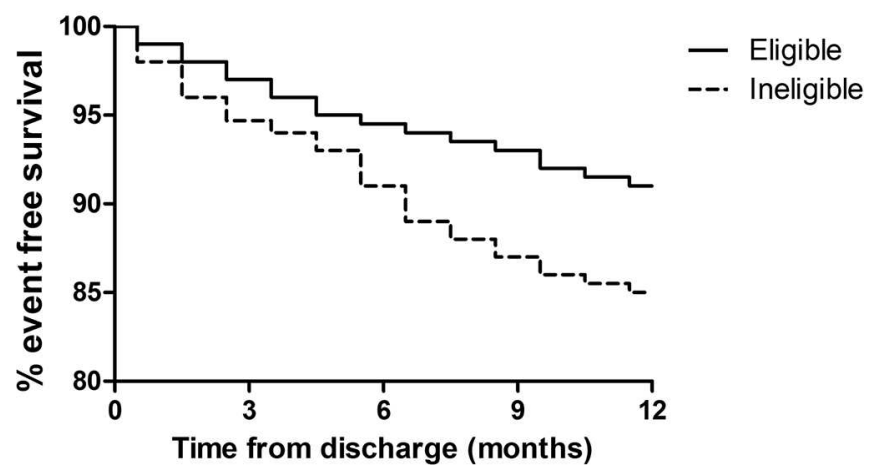

Abstract P224 Figure 1.

\section{P225 INHALER USE AND MISUSE ON THE WARDS OF HOSPITAL}

'D Long, ${ }^{2}$ RB Reilly; ${ }^{1}$ Royal College of Surgeons in Ireland, Dublin, Ireland; ${ }^{2}$ Trinity College Dublin, Dublin, Ireland

\subsection{6/thoraxjn-2013-204457.377}

Clinicians are aware that inhalers are often improperly used incorrectly by patients. However, it often difficult to assess, because at present there is no tool that directly quantify adherence. We designed a device, the INCA device that makes an acoustic recording each time an inhaler is used. Opening the device starts the recording, this electronic sound file is "timestamped" which means that the timing of drug administration is recorded, while analysis of the acoustics identifies the technique of inhaler use. When the INCA device is retrieved and acoustic analysis performed, the steps involved in using the inhaler are determined. Hence, both inhaler technique and the time when the inhaler was used can be identified. Together this means gives an objective quantitative assessment of inhaler adherence.

In this study we attached the INCA device to a diskus dry powder inhaler and studied inhaler use by people in Hospital who were prescribed a diskus inhaler.

Initial results from three general Hospitals $(\mathrm{n}=50)$ indicated that there were errors in both overuse $15 \%$ of doses, missed doses in $30 \%$ of patients and poor inhaler technique was seen in $45 \%$ of patients, in no case was it suggested that the device be changed. Overall, $<40 \%$ of inhaler doses were administered on time and in the correct manner. Investigation indicated that inhaler administration was not supervised which together meant that errors in inhaler use were not rectified. Subsequently we undertook an institution wide comprehensive practice change involving prescription review, changes to storage and administration policy, supervised inhaler administration to correct inhaler misuse and a follow-up when inhaler misuse persisted despite ward level instruction. Six months after the introduction of practice change a series of follow on audits were performed. One audit indicated that the storage and administration practice was adopted on the wards. The second indicated in 100 consecutive admissions that observed inhaler technique was adequate in $33 \%$ of patients on admission, improved in 33\% and was unchanged by discharge in 33\%. Thirdly, studies with the INCA device, $(n=40)$ indicated that overdosing was documented in only $2 \%$ of recordings, missed doses were reduced to $20 \%$ and $10 \%$ of patients were changed from one device to a more suitable one. Hence, data from the INCA device prompted a change in practice Improvements in inhaler use on the wards were achieved by a simple quality improvement intervention.

\section{P226 INHALATION CHARACTERISTICS WITH SPIROMAX ${ }^{\circledR}$ AND TURBUHALER ${ }^{\circledR}$ DRY POWDER INHALERS (DPI) IN HEALTHY ADULTS AND PATIENTS WITH ASTHMA OR CHRONIC OBSTRUCTIVE PULMONARY DISEASE (COPD): BEFORE AND AFTER ENHANCED TRAINING}

${ }^{1} \mathrm{H}$ Chrystyn, ${ }^{1} \mathrm{~W}$ Azouz, ${ }^{2} \mathrm{P}$ Chetcuti, ${ }^{3} \mathrm{H}$ Hosker, ${ }^{4} \mathrm{D}$ Saralaya; ${ }^{1}$ University of Huddersfield, Huddersfield, United Kingdom; 'Leeds General Infirmary, Leeds, United Kingdom; ${ }^{3}$ Airedale General Hospital, Bradford, United Kingdom; ${ }^{4}$ Bradford Royal Infirmary, Bradford, United Kingdom

\subsection{6/thoraxjn-2013-204457.378}

Introduction and Objectives Acceleration of inhaled flow from a DPI is important to facilitate de-aggregation of the metered dose and to ensure delivery of an appropriate dose. Patients need to inhale as fast as possible from the beginning of their inhalation manoeuvre and continue inhaling until their lungs are full. ${ }^{1}$ This study investigated inhalation characteristics when patients and healthy adults (HA) inhale through placebo Spiromax ${ }^{\circledR}$ and placebo Turbuhaler ${ }^{\circledR}$ DPIs and assessed the impact of enhanced DPI technique training.

Methods This was a randomised, open-label, crossover study involving children (6-11 years old, [CA]), adolescents (12-17 years old [AA]) and adults with asthma [ADULT], COPD patients and HA. Study participants were trained to use the Spiromax and Turbuhaler DPIs according to the Patient Information Leaflets. Inhalation characteristics were measured.

Each participant received enhanced training using an InCheck Dial $^{\mathrm{TM}}$ to measure inspiratory flow (IF). Participants were encouraged to increase their IF by inhaling more quickly. Inhalation characteristics were measured in the same way as before enhanced training.

Results Before enhanced training,peak inspiratory flow (PIF) and maximum change in pressure $(\mathrm{P})$ were significantly higher with Spiromax versus Turbuhaler ( $\mathrm{p}<0.05$; all patient groups). There were also trends towards slightly higher inspiratory acceleration (ACC) with Spiromax.

Table 1 shows the pre-training inhalation characteristics. Enhanced training significantly improved PIF, ACC and P ( $\mathrm{p}<$ 0.05 ) in all subjects and in both inhalers except $P$ with Spiromax in patients with COPD. Percentage improvements in P and ACC are shown in Table 1 . Significantly greater improvements $(\mathrm{p}<$ 0.05 ) were seen with Spiromax versus Turbuhaler (post training) for PIF (all groups), P in AA, ADULT and patients with COPD, and for ACC in ADULT and COPD patients.

Conclusions Patients achieved faster IF and greater positive change in pressure with Spiromax versus Turbuhaler. Enhanced 